\title{
A randomised controlled intervention trial evaluating the efficacy of an Australianised Mediterranean diet compared to the habitual Australian diet on cognitive function, psychological wellbeing and cardiovascular health in healthy older adults (MedLey study): protocol paper
}

Courtney R. Davis ${ }^{1 *}$, Janet Bryan², Jonathan M. Hodgson ${ }^{3}$, Carlene Wilson ${ }^{4}$, Varinderpal Dhillon ${ }^{5}$ and Karen J. Murphy ${ }^{1}$

\begin{abstract}
Background: The Mediterranean diet (MedDiet) is associated with considerable health benefits for cardiovascular and cognitive health, particularly within the Mediterranean basin. Whether Australian populations will similarly benefit from the MedDiet is unknown. We aim to assess the effects of a MedDiet on cognitive and cardiovascular health indicators amongst the elderly (MedLey study). Here we describe in detail the protocol for the MedLey study.

Methods: Medley is a randomised, parallel controlled dietary intervention trial. Data collection occurred at the Sansom Institute for Health Research, University of South Australia, Adelaide, South Australia, Australia between 2013 and 2015. Omnivorous men and women aged $\geq 65$ years, who were non-smokers, had no previously diagnosed cardiovascular disease, history of brain trauma, cognitive impairment, major disease or cancer were recruited from the general public $(n=166)$. The intervention comprised an Australianised version of the traditional MedDiet characterised by small amounts of red meat, processed deli meats and high sugar foods; moderate amounts of dairy and poultry; and a high plant food content including abundant extra virgin olive oil. Volunteers were stratified by age, gender and body mass index $\left(\mathrm{kg} / \mathrm{m}^{2}\right)$ to follow either their habitual diet (control condition) or the intervention MedDiet for 24 weeks. Primary outcome measures included cognitive function, endothelial function, cerebrovascular flow, blood lipids, inflammatory markers, fasting glucose and insulin, blood pressure, and body composition. Secondary outcomes included food cravings, psychological wellbeing and DNA damage markers. These were assessed at baseline, 12 and 24 weeks. Dietary intakes were assessed at baseline, two and four months to assess adherence to the prescribed diet. For main outcome measures, groups will be compared over time using a linear mixed effects model, with Cohen's d effect sizes. Subgroup analysis based on adherence to the intervention, medications and alcohol consumption may be performed in an exploratory fashion.

(Continued on next page)
\end{abstract}

\footnotetext{
*Correspondence: courtney.davis@mymail.unisa.edu.au

${ }^{1}$ Alliance for Research in Exercise, Nutrition and Activity, University of South

Australia, GPO Box 2471, Adelaide, South Australia 5001, Australia

Full list of author information is available at the end of the article
} 
(Continued from previous page)

Discussion: The MedLey study will provide evidence as to whether dietary changes in line with an Australianised MedDiet can benefit cognitive performance, endothelial function and other cardiovascular risk factors and psychological wellbeing amongst older Australians. Results could inform formation of national dietary guidelines and nutrition-related practices such as dietetics.

Trial registration: The trial was registered on the Australia New Zealand. Clinical Trials Register (ACTRN12613000602729). Trial registration date: 27 May 2013.

Keywords: Mediterranean diet, Cognitive function, Cardiovascular health, Randomised trial, Australia

\section{Background}

Several observational studies have supported the Mediterranean dietary pattern as a healthy diet that lowers risk of cardiovascular disease (CVD) [1]. Cardiovascular diseases are responsible for nearly one fifth of total deaths in Australia, occurring mostly as coronary heart disease (myocardial infarction and angina) [2]. The precursor is atherosclerosis, an inflammatory condition affecting the functional capacity of the blood vessels $[3,4]$. The exact mechanism(s) involved in the development of atherosclerosis are still being understood, however, the link between various lifestyle factors such as smoking, physical activity and diet and the prevalence of CVD have been well established through epidemiological studies $[5,6]$. Those consuming a plant-based diet (fruits, vegetables, nuts, seeds and vegetable oils) have a reduced risk of CVD [7]. The diets followed traditionally in Mediterranean countries including Greece, Italy, Spain and France include these components and have been repeatedly linked to improvements in cardiovascular health indicators, and reduced mortality rates from CVD [8-11]. A metaanalysis of observational trials showed higher adherence to the MedDiet reduces risk of CVD including coronary heart disease and stroke, as well as overall mortality [1]. Recently, the PREDIMED (Prevención con Dieta Mediterránea) study, a large scale randomised controlled trial, reported that this dietary pattern supplemented with extra virgin olive oil or nuts, reduces the risk of major cardiovascular events in individuals at high CVD risk [12]. The Mediterranean diet (MedDiet) is also consistent with the Australian Heart Foundation's recommendations for a healthy diet, that is high in fibre, omega-3 long chain fatty acids, plant sterols, and low in saturated and trans fatty acids [13-15]. The effect of the MedDiet on cardiovascular biomarkers and end-points has previously been tested in European populations with positive outcomes $[11,12,16]$. However, only one small $(n=20)$ randomised cross-over trial has been conducted in Australia on markers of diabetes severity [17]. With promising results in other countries, studies are needed to determine if this dietary pattern is a viable option to improve markers of cardiometabolic health and potentially reduce incident CVD in non-Mediterranean populations like Australia. The following will describe the methods for the Mediterranean diet for cognitive and cardiovascular health in the elderly (MedLey) study.

\section{Methods}

Aim

We aim to determine the effects of an Australianised version of the traditional MedDiet on cardiometabolic biomarkers and cognitive performance. The secondary aim is to determine how closely Australians can adhere to Mediterranean dietary practices with dietetic supervision. This paper presents the protocol for the recruitment and randomisation, study design, methods of dietary, cognitive and cardiometabolic data collection and describes the Australianised MedDiet and the measures of dietary compliance used.

\section{Recruitment and randomisation}

Volunteers were recruited from metropolitan Adelaide, South Australia, Australia. Exclusion criteria are shown in Table 1. These criteria were assessed by a diet and lifestyle questionnaire (DLQ) used to screen volunteers expressing interest. If eligible based on the DLQ, volunteers were screened at the Sansom Institute for Health Research clinic (University of South Australia, Adelaide) for evidence of cognitive decline (assessed by the DemTect screening tool for the presence of dementia and mild cognitive impairment) [18] and had their fasting blood lipids, glucose, insulin, high sensitivity C-reactive protein ( $h s$-CRP), liver function and immune function assessed by a full blood examination to detect health issues.

Additionally body mass, height and blood pressure were measured to confirm eligibility. A rolling recruitment occurred in two cohorts, the first from June to September 2013 and the second from March to May 2014. Seventyseven volunteers were enrolled for cohort one and 89 for cohort two. The staggered-start method was used. A maximum of 10 volunteers were started per week for 12 weeks, with an interval of three weeks to avoid scheduling visits during the Christmas holiday period. Baseline data collection occurred between August and November 2013 for cohort one, and between May and August 2014 for cohort two. Fourteen volunteers withdrew before commencement, and baseline data was collected for 152 volunteers (Fig. 1). 
Table 1 Exclusion criteria

- Person considered by the investigator to be unwilling, unlikely or unable to comprehend or comply with the study protocol

- Age $<65$ years

- Previous/current traumatic head/brain injury, neurological or psychiatric conditions

- Previous stroke

- Unstable use of anti-depressant medication ( $<6$ months)

- Use of medication to treat anxiety

- Current or recent (in the last 6 months) malignancy

- Major liver, kidney, respiratory or gastrointestinal disease

- Current cardiovascular disease or angina

- Uncontrolled hypertension (>170/100)

- Current smoker

- Vegetarian (does not eat red meat, poultry or fish)

- Actively undertaking weight loss program

- Use of appetite suppressants or Orlistat (Xenical)

- Age and education adjusted scores of $<13$ on the DemTect

- Body mass $>135 \mathrm{~kg}$ (limit on dual xray energy absorptiometry (DEXA) scanner)

Volunteers were randomly allocated to either the habitual diet (HabDiet) control group or the MedDiet intervention group stratified by gender, body mass index (BMI) and age by the process of minimization [19]. An honorarium payment of $\$ 100$ was awarded to volunteers who commenced the study on a pro rata basis to cover

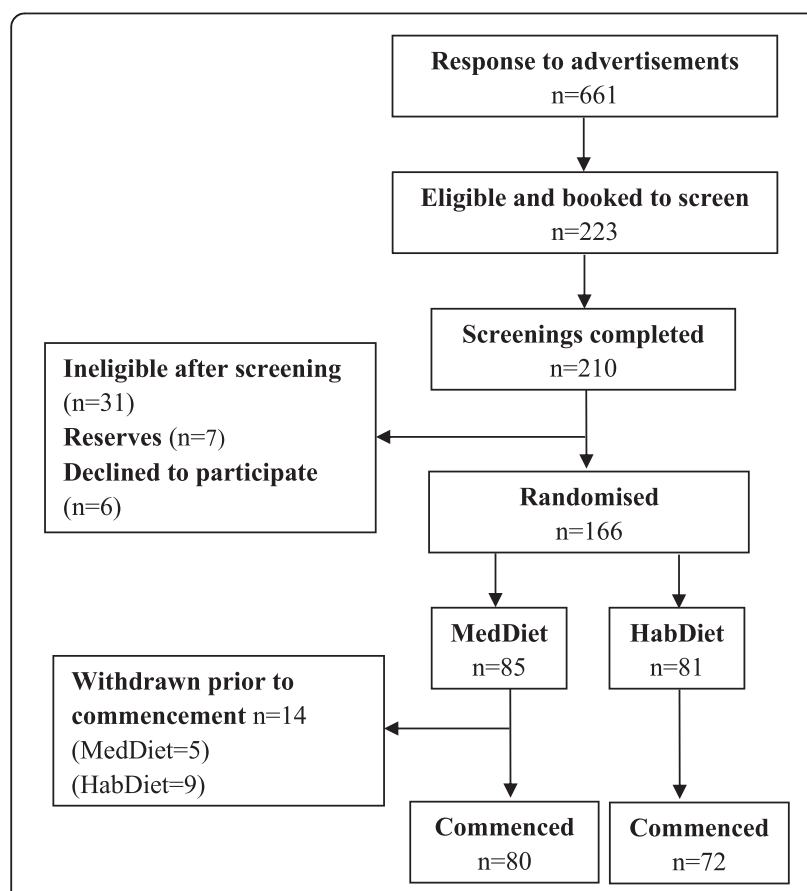

Fig. 1 Consort flow diagram for recruitment, randomisation and baseline data collection parking and travel related expenses. The study was conducted according to the guidelines laid down in the Declaration of Helsinki and all procedures involving human participants were approved by the Human Research Ethics Committee (22 June 2013, \#31163), University of South Australia, Adelaide, Australia. Written informed consent was obtained from all participants before commencement.

\section{Study design}

MedLey is a randomised, controlled dietary intervention trial lasting 24 weeks, including six major and 10 minor clinic visits all of which occurred at the Sansom Institute for Health Research. Volunteers first attended two clinic visits spaced 1 week apart during which primary and secondary outcome measures were assessed to obtain baseline data (Fig. 2). Primary outcome measures included cognitive function, endothelial function, cerebrovascular flow, blood lipids, inflammatory markers, fasting glucose and insulin, blood pressure, and body composition. Secondary outcomes included food cravings, psychological wellbeing and DNA damage markers. Additionally dietary intake was assessed. These assessments are described in detail in following sections. Both baseline visits were conducted after a minimum $8 \mathrm{~h}$ fast (water allowed). At the first clinic visit, (Visit 1A), a $26 \mathrm{ml}$ blood sample was collected to measure blood lipids, glucose, insulin, $h s$-CRP, erythrocyte fatty acids, $\mathrm{F}_{2}$-isoprostanes and carotenoids. A buccal cell, saliva and $9 \mathrm{~mL}$ blood sample was also provided by a sub-sample of participants not taking any medications, for assessment of the $\mathrm{ApoE}_{4}$ genotype and DNA damage biomarkers (telomere length, mitochondrial DNA deletions, DNA base oxidation and micronucleus index). Blood pressure, trans cranial Doppler (TCD), flow mediated dilatation (FMD) and cognitive function was also assessed at Visit 1A. During the following six days volunteers collected a 24-hour urine sample, completed a 3-day weighed food record (WFR) and monitored their blood pressure. A series of questionnaires to assess psychological wellbeing and a validated food frequency questionnaire (FFQ) were completed $[20,21]$. Volunteers were also administered a demographic questionnaire, collecting information on country of birth, marital, educational and work status and level of income. Upon return to the clinic (Visit 1B) these questionnaires, the blood pressure monitor and readings, urine sample and 3-day WFR were returned. Height, body mass, waist and hip circumference, bone mineral density and body composition (by dual $\mathrm{x}$-ray absorptiometry (DEXA) scan) were assessed and the volunteers received their dietary instructions. Excluding the WFR, FFQ and demographic questionnaire, these assessments were undertaken again after 12 and 24 weeks ( 3 and 6 months). Totally, the study period lasted 25 weeks (from week $0-1$ between Visit $1 \mathrm{~A}$ and Visit1B volunteers were not following their allocated diets). Volunteers attended 10 minor clinic visits 


\begin{tabular}{|c|c|c|}
\hline $\begin{array}{l}\text { Week A Clinic visit } \\
\text { - Fasting blood } \\
\text { sample } \\
\text { - Blood pressure } \\
\text { - FMD } \\
\text { - TCD } \\
\text { - Cognitive } \\
\text { assessments }\end{array}$ & $\begin{array}{l}\text { During week } \\
\text { - FFQ and 3-day WFR (baseline clinic } \\
\text { visits only) } \\
\text { - Demographic questionnaire } \\
\text { - Food craving inventory } \\
\text { - } \text { Psychological wellbeing questionnaires } \\
\text { - SF-36v2 Health survey } \\
\text { - Daily blood pressure } \\
\text { - } 24 \text { hour urine sample collection }\end{array}$ & $\begin{array}{l}\text { Week B Clinic visit } \\
\text { - Fasting DEXA } \\
\text { scan } \\
\text { - Body mass and } \\
\text { height } \\
\text { - Waist and hip } \\
\text { circumference } \\
\text { - Diet consultation }\end{array}$ \\
\hline
\end{tabular}

Fig. 2 Assessments at each clinic visit

fortnightly, each approximately $30 \mathrm{~min}$. These visits engaged volunteers with a dietitian to discuss the dietary component of the trial, report changes in medications and physical activity levels and any adverse events. A lifetime diet questionnaire (LDQ) was administered during week 21 (cohort 1) or week 5 (cohort 2) to capture information on eating habits throughout the lifespan [22]. A follow-up FFQ and 3-day WFR were administered at week 11 and week 19. These were completed during the fortnight and returned at the subsequent appointment.

A protocol amendment occurred during baseline data collection of cohort one. It was noted that volunteers were commenting on their experiences with food cravings. It was decided that the Food Cravings Inventory (FCI) [23] should be administered at each time point to formally capture changes related to food cravings. This was altered in the protocol and included as an ethics amendment. Therefore excluding withdrawals, 16 volunteers from cohort one and all volunteers from cohort two $(n=74)$ completed the FCI at all visits. A further 10 volunteers completed the FCI at baseline and 6 months in cohort one.

\section{Assessments}

\section{Cognitive and psychological well-being assessments}

The selected cognitive test battery was designed to assess cognitive performance indicators sensitive to aging and nutritional factors, based on published literature. The battery assessed verbal and spatial episodic memory, verbal working memory, high order executive functions, attention and speed of processing, and is described in detail in Knight et al. [24]. Briefly, the cognitive test battery included the following (in order of presentation to the participants): D-KEFs Tower of London, the Rey Auditory Verbal Learning Test, the WAIS IV Symbol Search and the WAIS IV Coding, the Stroop test, the WAIS-IV digit span forward, digit span backwards and letter-number sequencing, Initial Letter Fluency, Excluded Letter Fluency, and the Benton Visual Retention Test. Alternate forms of these tests were presented to individuals at each time point in order to reduce practice effects. The sequence of alternative versions was randomly determined by a researcher not involved in data collection. Alternate sequences were counter-balanced across all visits. All cognitive testing took place in the same private clinic room after an $8 \mathrm{~h}$ fast. Wherever possible, the tests were administered at the same time of day and by the same researcher at each visit. Scoring of the test battery was completed by the same researcher for all participants.

To assess psychological wellbeing, the following questionnaires were completed during the week between visit A and visit B: the SF-36 Health Survey (version 2) [25], the Self-evaluation Questionnaire (STAIP-AD test form Y-1 and Y-2) [26], the Centre for Epidemiological Studies Depression Scale (CES-D) (NIMH) [27], the Perceived Stress Scale [28], and the Leeds Sleep Evaluation Questionnaire [29]. These assessments were completed again at 3 and 6 months.

\section{Cardiometabolic measures Trans cranial Doppler}

TCD is non-invasive measure of cerebrovascular responsiveness (CVR) in the middle cerebral artery (MCA) and has been described elsewhere [24]. Briefly, participants sat in a temperature-controlled room $\left(22^{\circ} \mathrm{C}\right)$ for at least 10 min prior to commencing the assessment. The transtemporal window was used as the acoustic window for insonation of the MCA at a mean depth of $50 \pm 10 \mathrm{~mm}$. Where possible, a clear signal for the left and right side was obtained - when not possible, one side was still recorded and included in analysis. If neither left nor rightside trans-temporal windows gave a clear signal, after approximately $10 \mathrm{~min}$ of searching the test was abandoned and not repeated at subsequent visits. Participants were fitted with a non-rebreathing two-way valve and facemask encompassing nose and mouth (General Electric Logiq 5 Expert). A $30 \mathrm{~s}$ baseline clip was recorded to establish normal flow velocity. The participant then inhaled carbogen gas $\left(5 \% \mathrm{CO}_{2} / 95 \% \mathrm{O}_{2}\right)$ for $150 \mathrm{~s}$ to initiate hypercapnia induced cerebrovascular dilatation. Mean flow velocity in the MCA was recorded for each cardiac cycle. To improve test reliability, this assessment was repeated three times with a rest period of at least $2 \mathrm{~min}$ between tests to allow flow velocity to return to baseline 
levels. Analysis was performed using Systat Software Inc. (San Jose, California, USA) and the peak increase in mean flow velocity will be determined. CVR will be expressed as a percentage change from baseline [(peak mean flow velocity - baseline mean flow velocity) $* 100$ /baseline mean flow velocity].

\section{Flow mediated dilatation}

The primary cardiovascular health outcome measure of MedLey is endothelial function, assessed by FMD of the right-side brachial artery. FMD was measured using the techniques described by Stout [30]. The volunteer was positioned supine on an electronically height-adjustable bed, in a temperature controlled room $\left(22^{\circ} \mathrm{C}\right)$. The bed height was adjusted so that the volunteer's right arm could be extended horizontally on the same vertical plane of the heart. The upper arm and hand rested on pillows, with space around the forearm to allow for positioning and inflation of a blood pressure cuff. The cuff was placed $2-3 \mathrm{~cm}$ below the antecubital fossa. The volunteer was at rest for a minimum of $10 \mathrm{~min}$ before commencement, and had abstained from all food and drink (other than water) for a minimum of $8 \mathrm{~h}$. A twodimensional B-mode $12 \mathrm{MHz}$ transducer (General Electric Logiq 5 Expert) ultrasound probe was used at a depth of $2 \mathrm{~cm}$. The probe was placed 3-7 $\mathrm{cm}$ above the antecubital fossa, after palpating for the location of the brachial artery. Three five-second recordings, spaced at least five seconds apart, were recorded before inflation to determine baseline artery diameter ( $15 \mathrm{~s}$ in total). The blood pressure cuff was then rapidly inflated to $200 \mathrm{mmHg}$ to occlude blood flow for $5 \mathrm{~min}$. A $5 \mathrm{~s}$ recording was taken at $4: 45 \mathrm{~min}$ to determine predeflation diameter. At $5 \mathrm{~min}$, the cuff was rapidly deflated and the images continuously recorded for three minutes post deflation, in $15 \mathrm{~s}$ intervals. The electrocardiogram for all volunteers was recorded with all videos. Analysis will be performed using the Vascular Tools for Research software package, Brachial Analyser for Research with DICOM module (Medical Imaging Assessment, version 6.1.3).

As fewer participants were needed to meet power for TCD and FMD than the total recruited, only participants with high quality scans had TCD and FMD performed at all time-points. To determine scan quality, a grading system was developed by JH, CD, KM and JB. For FMD, scores were based on the following criteria: number of images recorded in full, visibility of the intima/media layers in all clips, and extent of movement of the ultrasound probe, especially between baseline and postdeflation recordings. Other considerations included participant movement and discomfort. A similar scoring system was utilised for TCD, criteria including poor signal strength, volunteer intolerance and movement and poor image clarity. Where possible, all participants underwent FMD and TCD at baseline, and each scan was graded immediately by the operator from 1 to 5 . A grade of five reflected maximum quality. If the scans were considered unsuitable due to any of the criteria not being met, a rating of 3.5 or less was given. Only baseline scans with ratings between 3.5 and five were repeated at subsequent visits. Complete TCD data was obtained at three visits for 71 volunteers, and a further 15 had data from two visits. FMD scans were complete for 96 volunteers at three visits, and a further six had data for two scans. Independent samples t-tests were used to determine whether there were any differences between those with complete data and those without for age, BMI, waist to hip ratio, body fat $\%$ and blood lipids at baseline. For TCD, total cholesterol was significantly higher amongst those without scan data $(P<0.05)$, and for FMD total cholesterol and LDL cholesterol were significantly higher amongst those without scan data $(P<$ 0.01 , respectively). Chi squared tests showed there was no relationship between gender $(P>0.5)$ or diet allocation and whether TCD was performed $(P>0.5)$, and no relationship between gender $(P>0.2)$ or diet allocation and whether FMD was performed $(P>0.2)$ indicating groups were even for gender and diet allocation.

\section{Blood and urinary parameters}

Trained staff used venepuncture to collect samples using the Vacuette ${ }^{\oplus}$ blood tube collection system. Samples were collected at clinic visit $\mathrm{A}$ at each time point for analysis of triglycerides, total cholesterol, low-density lipoprotein (LDL), high-density lipoprotein (HDL), and total cholesterol to HDL ratio, glucose, insulin, hs-CRP, erythrocyte fatty acids (FAs), $\mathrm{F}_{2}$-isoprostanes and carotenoids. After collection blood tubes were immediately placed on ice and shielded from light. Lipids, glucose, insulin and hsCRP were analysed at a National Association of Testing Authorities (NATA) accredited pathology centre within two hours of collection.

Blood samples for the remaining measures were centrifuged at $4000 \mathrm{rpm}$ for $10 \mathrm{~min}$ at $4{ }^{\circ} \mathrm{C}$ and stored at $-80{ }^{\circ} \mathrm{C}$ until analyses. Carotenoids, retinol and tocopherols were measured from plasma according to the method of Barua et al. [31] using high performance liquid chromatography. Erythrocyte fatty acids were measured using direct transesterification as described elsewhere [32].

Volunteers collected a $24 \mathrm{~h}$ urine sample for analysis of 24-hour excretion of urinary magnesium, calcium, potassium and sodium, to measure dietary adherence. Sample collection started after completion of the first void on the morning before, and continued up to and including the first void on the morning of the second clinic visit (Visit B). Urinary metabolites (sodium, potassium, 
magnesium and calcium) were analysed at a NATA accredited lab.

\section{Assessment of DNA damage biomarkers and $\mathrm{ApoE}_{4}$ genotyping}

For the assessment of DNA damage and $\mathrm{ApoE}_{4}$ genotyping, a sub-group of volunteers $(n=22)$ who were not taking medication were selected. Data was collected at baseline and visit 3A for 20 volunteers (eight males, 12 females, eight MedDiet, 12 HabDiet). For DNA isolation, a $2 \mathrm{ml}$ saliva sample was collected using the Oragene DNA kit (OG-500, DNA Genotek), and a buccal cell sample was provided. In the fasted state, volunteers rinsed their mouths with water thoroughly then filled a $2 \mathrm{ml}$ collection tube with saliva. This was then immediately mixed with a stabilisation reagent and stored at room temperature until analysis. Volunteers used soft-bristled brushes to obtain buccal cells from the left and right side cheek. After collection, brushes were immediately washed in a stabilisation reagent and stored at room temperature. ApoE4 genotyping was performed using the TaqMan ${ }^{\odot}$ SNP genotyping assay kit (Applied Biosystems) as described by Koch et al. [33].

\section{Cytokenesis-block micronucleus-cytome assay}

A $9 \mathrm{ml}$ blood sample was collected for the Cytokenesisblock micronucleus-Cytome (CMBN-Cyt) assay [34]. Heparinised blood was collected and plasma separated by centrifugation. Plasma was removed and lymphocytes were isolated as described previously by Fenech et al. [34]. The CBMN-Cyt assay is a validated measure of chromosomal DNA damage, cytostasis, and cytotoxicity [34]. The degree of chromosomal DNA damage can be quantified by scoring the frequency of bi-nucleated cells (cells with two nuclei) containing micronuclei, nucleoplasmic bridges, and nuclear buds. Cytostasis was determined by calculating the nuclear division index (NDI) using ratios of viable cells with one, two, or more than two nuclei whereas cytotoxicity was gauged by the relative ratio of dead (apoptotic or necrotic) to viable cells. Cytostasis was determined by the NDI, calculated by scoring the ratio of viable mono-nucleated, bi-nucleated, and multi-nucleated (three or more nuclei) cells per 500 cells according to the following formula: $\mathrm{NDI}=\left(\mathrm{M}_{1}+\right.$ $\left.2 \mathrm{M}_{2}+3 \mathrm{M}_{3}+4 \mathrm{M}_{4}\right) / \mathrm{T}$ where $\mathrm{T}$ is total number of viable cells scored). Cytotoxicity was determined via the number of apoptotic and necrotic cells scored per 500 cells, whereas chromosomal DNA damage was measured by scoring the number of micronuclei nucleoplasmic bridges and nuclear buds per 1000 BN cells. The scoring criteria are as previously described for lymphocytes [34].

\section{Telomere length measurement}

Both relative repeat copy number of telomere $(\mathrm{T})$ and single copy gene (36B4) (S) were determined using real- time polymerase chain reaction (PCR) as described previously [35] with power SYBR green PCR master mix using Applied Biosystems 7300 Real-Time PCR System (Applied Biosystems, USA). Each sample was analysed in triplicate both with telomere and 36B4 gene primers. To perform standard curves with each gene, one reference DNA was serially diluted with deionised water by 1.68 fold per dilution to create seven concentrations of DNA ranging from 1.78 to $40 \mathrm{ng} / \mu \mathrm{L}$. The $\mathrm{R} 2$ of the standard curves were $>98.5 \%$, and the amplification efficiency of each pair of primers was $>98.5 \%$. The relative telomere length was expressed as the ratio of these two values ( $\mathrm{T} /$ $\mathrm{S}$ ratio), reflecting the average telomere repeat copy number of each DNA sample calculated relative to reference DNA. To control for inter-assay variability, five control DNA samples were also included in each PCR run. We also used the absolute method as described previously [36] to calculate the actual telomere length in base pair in each individual.

\section{Anthropometry and body composition}

Height and body mass were measured to calculate BMI $\left(\mathrm{kg} / \mathrm{m}^{2}\right)$. Height was measured to the nearest $0.1 \mathrm{~cm}$ whilst barefoot using a wall-mounted stadiometer (SECA; Vogel and Halke, Hamburg, Germany). Body mass was measured to the nearest $0.1 \mathrm{~kg}$ with participants wearing a light hospital gown, using the TANITA Ultimate Scale 2000 (Tanita Corporation, Tokyo, Japan) after an eight hour fast. In cases where body mass could not be measured in a gown, volunteers were asked to remove shoes, heavy jewellery and outer layers of clothing and empty pockets. Body mass was also measured fortnightly at approximately the same time of day, without shoes and after removal of pocket contents and outer layers of clothing. Waist and hip measurements were taken using a Luftkin Executive Thin line $2 \mathrm{~m}$ metal measuring tape according ISAK international guidelines, as described by Norton and Olds [37]. Two measures of waist and hip circumference were taken to the nearest $0.1 \mathrm{~cm}$, and the average calculated. If the two measures differed by $2 \%$ or more a third measure was taken and the average of all three measures calculated. Waist circumference was measured directly over skin or over a thin layer of clothing. In cases where a narrowing of the waist was visible and lay between the tenth rib and top of the iliac crest, visual narrow was used. In cases where there was no visual narrowing, the mid-point between the tenth rib and the top of the iliac crest was used. Either mid-point or visual narrowing was used at all subsequent visits. Hip circumference was measured over the hospital gown or underwear, at the point of greatest protrusion of the gluteal. Waist to hip ratio was calculated by dividing waist circumference $(\mathrm{cm})$ into hip circumference $(\mathrm{cm})$.

Participants had their percentage of body fat, fat mass (kg), abdominal fat (g) and lean mass (\% and $\mathrm{kg}$ ) assessed 
using DEXA (Lunar Prodigy, General Electric, Madison, WI, USA) scans after an eight hour fast. Volunteers were required to void their bladder, remove shoes, outer clothing and jewellery, watches, glasses, and hearing aids. If it was not possible to remove all jewellery, any worn at baseline was also worn at subsequent scans. Wearing a hospital gown and underwear, the volunteer was instructed to lie supine on the DEXA bed, with palms flat and arms straightened by the sides. In cases where the hands were outside the scan area, volunteers were asked to place palms against thighs. Abdominal fat content was generated using Lunar Prodigy software from regional analysis of the DEXA scan to assess the region from the top of the iliac crest, with the lateral borders extending to the edge of the abdominal soft tissue, and the upper margin $20 \%$ above the pelvis between the pelvis and the neck borders.

\section{Blood pressure}

Blood pressure was assessed in clinic using an OMRON Healthcare Co. digital blood pressure monitor (model 1A1B (Hem-7000-CIL). Volunteers were seated for $10 \mathrm{~min}$, were asked to remove outer layers of clothing and/or roll up sleeves, and place feet flat on the ground. The forearm rested at table height, palm up, and the cuff was placed approximately $2-3 \mathrm{~cm}$ above the anti-cubital fossa. Three measurements of blood pressure were taken, each spaced at least one minute apart. Systolic and diastolic blood pressure and pulse were recorded. Volunteers were then provided with an A\&D Company ltd. digital blood pressure monitor (model UA-767), with detailed instructions to monitor their own blood pressure at home. Blood pressure was taken on six consecutive days, morning (prior to food or beverage consumption), during the afternoon and evening (before bed). At each time-point three readings of systolic, diastolic blood pressure and heart rate were recorded, each spaced at least one minute apart. Volunteers were instructed to avoid caffeine for $60 \mathrm{~min}$ and exercise for 30 min prior to taking the measurements.

\section{The Australianised Mediterranean intervention diet and measures of compliance \\ Development of the Australianised Mediterranean diet}

The MedLey diet was based on a literature review to determine the amounts of key foods and nutritional content of the MedDiet (unpublished work, Davis et al. 2015). Briefly, the review aimed to determine an average food and nutrient content, according to observations of Mediterranean populations and MedDiets used in previous intervention trials . Food content (in grams) was based on an average of 15 different MedDiets, and nutrient content on eight. The MedLey diet was modelled on these findings.

A recommendation for servings of 13 food groups was calculated based on the average gram content determined from the literature review. Serving sizes were based on current Australian guidelines, with several exceptions (eggs, wine, extra virgin olive oil (EVOO) and fish) [38]. A 7-day menu was then developed meeting the required numbers of servings per day and per week. The servings for fruits, nuts and dairy products had to be separately determined as they were not presented as isolated food groups in the literature review. Reports of traditional MedDiet intakes such as that in of the Cretan population reported in Kromhout et al. [39] were consulted as well as Mediterranean diet pyramids to determine an appropriate number of servings fruit, nuts, milk, cheese and yoghurt. The traditional Cretan diet included approximately $450 \mathrm{~g}$ fruit/day, not including nuts. For this reason the fruit recommendation was set at three $150 \mathrm{~g}$ servings per day. The recommendation for nuts was based on the Mediterranean diet foundation pyramid which suggests nuts/seeds should be consumed daily [40]. According to the literature review dairy foods comprised no more than one serve per day; however the Mediterranean diet foundation pyramid and reports of the traditional Cretan diet suggest a greater dairy consumption. Additionally, the Australian Nutrient Reference Values indicate the Estimated Average Requirement for calcium is 840$1100 \mathrm{mg} /$ day for an older population [41]. For these reasons it was deemed appropriate to increase the dairy content to 2-3 serves/day. To maintain the ratio of saturated to monounsaturated fatty acids skim milk and reduced fat yoghurt was strongly encouraged over full-fat versions. The final menu contained a standard number of servings for EVOO, vegetables, fruits, legumes, potatoes, wholegrain breads and cereal products, red wine, potatoes, yoghurt, fish, nuts, red meat and poultry.

The menu was entered into the dietary analysis software package FoodWorks Professional (Version 7.0.3016, Xyris Software Australia) to determine the nutrient content. This was compared with the nutrient content derived from the literature review. In cases where the nutrient content of the MedLey diet differed vastly from the average, key Mediterranean diet pyramids and papers outlining traditional intakes were consulted to ensure the diet reflected a traditional MedDiet. For example, fibre intake was averaged at $33 \mathrm{~g} /$ day in the literature review; however the total fibre was $47 \mathrm{~g} /$ day based on the menu, which is similar to traditional diets [39].

Because the traditional MedDiet is relatively high in energy (9300 kJ compared to average estimated requirement of $8700 \mathrm{~kJ})$, to account for lower energy requirements in the Australian population two lower energy versions of the diet were created. This was achieved by reducing the total numbers of servings of EVOO, vegetables, grain products, nuts, fruits, meats and extra foods. Energy levels 2 and 3 contained the same nutrients per $1000 \mathrm{~kJ} \pm 5 \%$ as the model diet (Energy level 1). The MedLey diet was then piloted over two weeks amongst 10 men and women aged $\geq 65$ years (Davis et al. under 
review). Several changes to the diet were introduced based on feedback from the pilot study including the creation of a 4th energy level $(6400 \mathrm{~kJ} /$ day $)$, the addition of recommendations for eggs, milk, smallgoods and extra (discretionary) foods, the introduction of flavoured Greek yoghurt as an alternative to plain, and a reduced recommendation for poultry. The MedLey diet menu was adjusted to incorporate these recommendations (Table 2). Either a minimum or maximum recommendation was given for each food group, with the exception of yoghurt and poultry where either exact requirements or a range was provided. For energy levels two and four ranges rather than minimums were also provided for fruit and EVOO to help control energy intake. Table 3 shows the nutrient content of each energy level.

\section{Measures of dietary intake}

Volunteers completed a 3-day WFR and FFQ at baseline, 2 and 4 months. Before completing the WFR, volunteers were instructed by trained personnel to include details of foods and beverages such as type (e.g., whether lowfat or full-fat), quantity (e.g., weight on packages, weight in grams, metric cup or spoon measures, or units of foods), method of preparation (e.g., fat trimmed, grilled, eaten raw), brand name and recipes of composite homecooked foods. All foods and beverages consumed in a consecutive 3-day period consisting of two week days and one weekend day were recorded. Volunteers were provided with digital kitchen scales to assist in accurately recording quantity. Clarification of any points of confusion was untaken by trained personnel at the time when the WFR was returned. FoodWorks Professional (Version 7.0.3016, Xyris Software Australia) was used to calculate nutrient and food intakes from WFRs. Volunteers were administered with the Cancer Council Victoria 74-item dietary questionnaire, validated for fruit and vegetable intake [21]. This was supplemented with additional questions regarding intake of soft drink, fish, nuts and oil. Volunteers were instructed to answer the baseline questionnaire based on the previous 12 months and the two and four month questionnaires on the previous two months only. Returned FFQs were checked for errors and accuracy with the volunteer.

\section{Administration of the Mediterranean diet and resources}

Volunteers were informed of their diet allocation at visit $1 B$ by a study dietitian. All study dietitians were trained by the chief dietitian in administration of the MedLey diet and HabDiet and were observed in their first 2-3 sessions with volunteers to ensure consistency in the delivery of the instructions. Volunteers commenced the diet the following day, and maintained this for the following 24 weeks.

Table 2 Recommended number of servings on the MedLey diet by energy level

\begin{tabular}{|c|c|c|c|c|}
\hline & Energy level 1 & Energy level 2 & Energy level 3 & Energy level 4 \\
\hline & $9600 \mathrm{~kJ} / 2300 \mathrm{kcal}$ & $8600 \mathrm{~kJ} / 2055 \mathrm{kcal}$ & $7400 \mathrm{~kJ} / 1770 \mathrm{kcal}$ & $6400 \mathrm{~kJ} / 1500 \mathrm{kcal}$ \\
\hline Foods (serving size ${ }^{a}$ ) & \multicolumn{4}{|l|}{ Daily servings } \\
\hline Extra virgin olive oil (1 Tbsp.) & $\geq 3$ & $2-3$ & $\geq 2$ & $1-2$ \\
\hline Vegetables (75 g) & $\geq 6$ & $\geq 5$ & $\geq 5$ & $\geq 5$ \\
\hline Fruits (150 g) & $\geq 3$ & $2-3$ & $\geq 2$ & $\geq 2$ \\
\hline Cereal products (30-120 g) & $\geq 6$ & $\geq 5$ & $\geq 4$ & $\geq 4$ \\
\hline Milk (250 ml) & $\leq 1$ & $\leq 1$ & $\leq 1$ & $\leq 1$ \\
\hline Red wine (150 ml) & $\leq 2$ & $\leq 2$ & $\leq 2$ & $\leq 2$ \\
\hline \multirow[t]{2}{*}{ Potato (white only) (1 medium) } & $\leq 1$ & $\leq 1$ & $\leq 1$ & $\leq 1$ \\
\hline & \multicolumn{4}{|l|}{ Weekly servings } \\
\hline Yoghurt (170 g) & 6 & 6 & 6 & 6 \\
\hline Cheese (40 g) & $\leq 4$ & $\leq 4$ & $\leq 4$ & $\leq 3$ \\
\hline Nuts (35 g) & $\geq 6$ & $\geq 5$ & $\geq 5$ & $\geq 4$ \\
\hline Legumes (75 g) & $\geq 3$ & $\geq 3$ & $\geq 3$ & $\geq 2$ \\
\hline Fish (150 g) & $\geq 3$ & $\geq 3$ & $\geq 3$ & $\geq 2$ \\
\hline Poultry (100 g) & $1-3$ & $1-3$ & $1-3$ & $1-3$ \\
\hline Red meat (100 g) & $\leq 1$ & $\leq 1$ & $\leq 1$ & $\leq 1$ \\
\hline Small goods (50 g) & $\leq 2$ & $\leq 2$ & $\leq 2$ & $\leq 1$ \\
\hline Eggs (1 egg) & $\leq 6$ & $\leq 5$ & $\leq 4$ & $\leq 4$ \\
\hline Extras (discretionary foods) (600 kJ) & $\leq 3$ & $\leq 3$ & $\leq 3$ & $\leq 2$ \\
\hline
\end{tabular}

${ }^{a}$ Serving sizes were loosely based on the Australian Guide to Healthy Eating standard serves, and were modified to increase similarity of the total food amounts to the MedDiet while retaining simplicity of the recommended numbers of serves for volunteers 
Table 3 Daily nutrient content of the Australianised Mediterranean diet, calculated from FoodWorks Professional ${ }^{a}$

\begin{tabular}{|c|c|c|c|c|}
\hline Nutrients & $\begin{array}{l}\text { Energy } \\
\text { level } 1\end{array}$ & $\begin{array}{l}\text { Energy } \\
\text { level } 2\end{array}$ & $\begin{array}{l}\text { Energy } \\
\text { level } 3\end{array}$ & $\begin{array}{l}\text { Energy } \\
\text { level } 4\end{array}$ \\
\hline Energy (kJ/kcal) & $9608 / 2300$ & $8593 / 2055$ & $7387 / 1770$ & $6384 / 1500$ \\
\hline Protein (g) & 97 & 90 & 79 & 64 \\
\hline Total fat (g) & 105 & 93 & 79 & 66 \\
\hline - SFA (g) & 20 & 18 & 15 & 11 \\
\hline - PUFA (g) & 20 & 16 & 14 & 13 \\
\hline - MUFA (g) & 58 & 53 & 44 & 37 \\
\hline Cholesterol (mg) & 90 & 83 & 80 & 66 \\
\hline $\mathrm{CHO}(\mathrm{g})$ & 209 & 186 & 158 & 146 \\
\hline Sugars (g) & 100 & 86 & 78 & 73 \\
\hline Alcohol (g) & 6 & 5 & 5 & 5 \\
\hline Dietary fibre (g) & 47 & 40 & 35 & 32 \\
\hline Vitamin C (mg) & 291.8 & 254.9 & 251.5 & 219.7 \\
\hline Vitamin E (mg) & 22.9 & 21.5 & 17.8 & 16.0 \\
\hline Total folate $(\mu \mathrm{g})$ & 529.9 & 454.7 & 440.4 & 391.7 \\
\hline $\begin{array}{l}\beta \text {-carotene } \\
\text { equivalents }(\mu \mathrm{g})\end{array}$ & 6141.9 & 5528.5 & 4988.6 & 4602.5 \\
\hline Sodium (mg) & 1887.8 & 1653.3 & 1315.5 & 1057.9 \\
\hline Potassium (mg) & 4324.5 & 3916.0 & 3574.0 & 3092.2 \\
\hline Magnesium (mg) & 451.8 & 408.1 & 354.0 & 306.7 \\
\hline Calcium (mg) & 841.3 & 783.9 & 652.4 & 571.2 \\
\hline Phosphorus (mg) & 1732.3 & 1594.3 & 1386.0 & 1137.9 \\
\hline Iron (mg) & 18.6 & 17.0 & 13.6 & 11.9 \\
\hline Zinc (mg) & 11.9 & 10.8 & 9.3 & 7.5 \\
\hline kJ from protein (\%) & 17 & 18 & 18 & 17 \\
\hline kJ from fat (\%) & 40 & 40 & 40 & 38 \\
\hline kJ from SFA (\%) & 8 & 8 & 8 & 7 \\
\hline kJ from MUFA (\%) & 22 & 23 & 22 & 21 \\
\hline kJ from CHO (\%) & 39 & 39 & 39 & 41 \\
\hline Fat as MUFA (\%) & 59 & 61 & 60 & 61 \\
\hline Fat as PUFA (\%) & 20 & 18 & 19 & 21 \\
\hline Fat as SFA (\%) & 21 & 21 & 21 & 19 \\
\hline $\begin{array}{l}\text { Long chain n-3 (EPA } \\
+ \text { DHA + DPA) (mg) }\end{array}$ & 1051 & 1045 & 1042 & 566 \\
\hline $\begin{array}{l}\text { 18:2n-6 linoleic } \\
\text { acid }(\mathrm{g})\end{array}$ & 16.94 & 13.32 & 12.19 & 11.17 \\
\hline $\begin{array}{l}\text { 18:3n-3 linolenic } \\
(A L A)(g)\end{array}$ & 1.78 & 1.38 & 1.08 & 1.11 \\
\hline
\end{tabular}

SFA saturated fatty acid, MUFA monounsaturated fatty acid, PUFA polyunsaturated fatty acid, $C H O$ carbohydrate, $n-3$ omega-3, EPA eicosapentaenoic acid, DHA docosahexaenoic acid, DPA docosapentaenoic acid, ALA alpha-linolenic acid

a Version 7.0.3016, Xyris Software Australia

For the HabDiet group instructions were to maintain current dietary intake, physical activity, dietary supplements and medications for the duration of the study, unless instructed otherwise by a medical professional.
Volunteers were informed that normal seasonal and occasional variations in diet were acceptable, and to avoid major changes such as introducing a new food or ceasing any regularly consumed food/s while on the study. The HabDiet volunteers were originally offered foods similar to those being provided to the MedDiet group (canola oil, yoghurt, bread and milk) to provide approximately $30 \%$ of estimated energy requirements per fortnight. The canola oil was kindly donated by Goodman Fielder Ltd. However, it was essential to avoid altering the diets of those randomised to the HabDiet. Due to the wide range of foods preferred by volunteers in the HabDiet, within the first fortnight it was deemed impractical to provide foods they usually consumed. Instead, we offered a \$10 (AUD) shopping voucher per fortnight for the volunteers preferred supermarket, allowing them to purchase up to $30 \%$ of their estimated energy requirements. This was amended in the protocol and approved by the ethics committee. Volunteers in cohort 1 continued to be offered foods in place of the voucher, however all accepted vouchers after the first fortnight. Volunteers in cohort 2 were not offered foods.

For volunteers on the MedLey diet, the instructions were given verbally with the aid of written resources which the volunteers kept for future reference. The education sessions lasted between $45 \mathrm{~min}$ and $1 \mathrm{~h}$. The volunteer first selected a day to represent a 'typical' day from their 3-day WFR completed the previous week. This was then entered into FoodWorks Professional by the dietitian aided by the volunteer to ensure accuracy. The energy intake calculated was then used to guide which of the four energy levels (9.6 MJ, 8.6 MJ, 7.4 MJ or $6.4 \mathrm{MJ} /$ day) of the MedLey diet was selected for the volunteer. Following this, volunteers had a short introduction to the fundamental principles of the MedDiet. Advice for recipe modification and eating out was given and volunteers received a recipe book. Volunteers were then presented with the serving sizes for each food group and given a table to indicate how many servings of key foods they had to consume, based on which energy level they were assigned (Table 2). Volunteers were encouraged to ask questions, such that the sessions were interactive. Finally, the daily checklists were provided and discussed to ensure the volunteer was competent at filling them out correctly. Volunteers were provided with Greek yoghurt, unsalted mixed nuts (peanuts, almonds, and walnuts), canned legumes, canned tuna and EVOO to last one fortnight. The amounts of these foods and their energy contributions for each energy level are shown in Table 4.

Volunteers purchased and prepared all other foods, and could consume the necessary servings at any time and in any way which suited them. Volunteers were instructed to maintain their current physical activity, dietary supplements and medications for the duration of 
Table 4 Foods provided to the Mediterranean diet group each fortnight and energy contribution to total

\begin{tabular}{|c|c|c|c|c|c|}
\hline \multirow[b]{2}{*}{ Foods } & \multirow[b]{2}{*}{$\begin{array}{l}\text { Amount provided } \\
\text { per fortnight }\end{array}$} & \multicolumn{4}{|c|}{$\%$ energy contribution to total fortnightly energy } \\
\hline & & Energy level $1(134,512 \mathrm{~kJ})$ & Energy level $2(120,302 \mathrm{~kJ})$ & Energy level 3 (103,418 kJ) & Energy level 4 (89,376 kJ) \\
\hline Legumes $^{a}$ & $450 \mathrm{~g}$ net weight & 1.5 & 1.6 & 1.8 & 2.0 \\
\hline Tuna $^{\mathrm{b}}$ & $190 \mathrm{~g}$ net weight & 0.6 & 0.7 & 0.8 & 0.9 \\
\hline Nuts $^{c}$ & Approximately $350 \mathrm{~g}$ & 8.1 & 7.6 & 7.0 & 8.1 \\
\hline Olive oil $^{d}$ & $750 \mathrm{ml}$ & 18.9 & 17.6 & 16.4 & 14.2 \\
\hline \multirow[t]{2}{*}{ Yoghurt ${ }^{\mathrm{e}}$} & $2.04 \mathrm{~kg}$ & 5.1 & 5.7 & 6.6 & 7.7 \\
\hline & Total \% energy provided & 34 & 33 & 33 & 33 \\
\hline
\end{tabular}

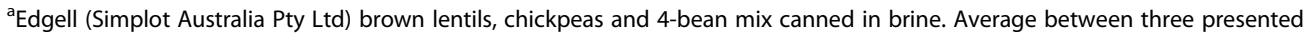

bJohn West (Simplot Australia Pty Ltd) plain, sweet chilli, lemon pepper and tomato onion in spring water

'Unsalted, raw nuts. Equal weights of peanuts, almonds and walnuts. Energy level 1 received $420 \mathrm{~g}$, energy level $2350 \mathrm{~g}$ and levels 3 and 4 received $280 \mathrm{~g}$ per fortnight

${ }^{\mathrm{d} C o b r a m ~ E s t a t e} \odot$ extra virgin olive oil, medium strength flavour. Volunteers received their daily quota as $750 \mathrm{ml}$ bottles (1 per fortnight), to provide 3 Tbsp/day. Thus energy levels 2, 3 and 4 did not use the whole quantity each fortnight

${ }^{\mathrm{e}}$ Chobani@ no fat plain, no fat flavours and $2 \%$ fat flavours provided. Volunteers were given 12 tubs weighing $170 \mathrm{~g}$ each per fortnight, and received an even mix of each type unless otherwise specified. An average of the three types is shown here

the study, unless instructed otherwise by a medical professional.

\section{Measures of compliance to the Mediterranean diet}

Compliance to the two study diets (MedLey and HabDiet) was monitored by measuring erythrocyte FAs (particularly MUFA:SFA), plasma carotenoids, urinary metabolites, FFQs and 3-day WFR's. Volunteers following the MedLey diet were also required to complete daily checklists to indicate they had consumed the required numbers of servings of foods. The foods listed on the checklist included EVOO, vegetables, fruits, wholemeal grain products, skim milk, potato, red wine, yoghurt, cheese, fish, nuts, legumes, red meat, poultry, smallgoods, eggs and extra foods (discretionary foods), for which there were recommended numbers of daily or weekly servings. Volunteers were instructed in detail by a trained dietitian to mark the checklist to indicate when one full serving was consumed. If more or less was consumed, volunteers were instructed to indicate this clearly. The volunteers returned the checklists every fortnight and these were discussed with a study dietitian to clarify serving sizes and address any issues or problems with compliance. Those following their habitual diets did not complete daily checklists.

\section{Power calculations}

A systematic review by Norman et al. [42] identified 38 studies which resulted in 62 effect sizes. For all but six studies the minimally important difference for healthrelated quality of life instruments was close to one half a standard deviation (mean $=0.495)$. Based on this effect size, we calculate that 128 subjects (two groups of $n=64$ ) will provide $80 \%$ power to detect a significant $(P<0.05)$ 0.5 SD difference in change in cognitive outcomes. A 0.5 SD improvement in cognitive outcomes is both clinically relevant and socially relevant as it would represent an annual cost saving of approximately $\$ 28$ million. An additional 38 volunteers (19 per group, total $n=166$ ) will be recruited to account for a $30 \%$ subject withdrawal rate. Generally the Sansom Research Institute has a less than $20 \%$ withdrawal rate. For FMD and TCD we calculate that 32 participants would give $80 \%$ power to detect a statistically significant $(P<0.05) 40 \%$ improvement, with an effect size of one in either FMD or CVR. A flow diagram of subjects' progress through the trial will be provided in accordance with the CONSORT statement.

\section{Statistics}

Descriptive statistics will be provided for the MedDiet and HabDiet groups for baseline characteristics, including clinical, sociodemographic and dietary information. For continuous variables, means and SD are provided. For categorical variables, counts and percentages are provided. To assess differences between groups in cognitive function and cardiovascular outcomes, a linear mixed effects model will be fitted. Non-normally distributed variables will be $\log$ transformed before analysis. Covariates will be determined using a correlation matrix to identify significant interactions between potential confounders. Outcomes will be adjusted for energy intake. An unadjusted and adjusted model will be presented. The unadjusted model will include age, gender and BMI where significant. The adjusted model will include these parameters as well as any potential confounders found to be correlated to outcomes which may include energy intake, demographic variables, physical activity, and psychological variables. We will analyse on an intention to treat basis. To assess effect size Cohen's d will be calculated. Subgroup analyses adjusting for dietary compliance, medication use and alcohol consumption may be performed in an exploratory capacity. Statistical significance will be set at $P \leq 0.05$, and analysis will be performed using SPSS for Windows (version 20.0; SPSS, Chicago, IL). 


\section{Discussion}

The MedLey study is designed to assess the effects of an Australianised MedDiet on cognitive function and cardiovascular health indicators over 6 months in a healthy population of older adults. This paper outlines the protocol for the collection of cardiometabolic and cognitive data, the design and administration of the MedLey diet and measurements of compliance.

So far no randomised trials in Australia have investigated any effect of the MedDiet on cardiometabolic risk factors for CVD without weight loss. The Medi-RIVAGE study was a 3 month randomised trial conducted in France comparing the effects of a modernised MedDiet with the low-fat diet recommended by the American Heart Association on clinical and biochemical risk factors for CVD [16]. Total and LDL cholesterol, triglycerides and glucose decreased in both groups, with a tendency for greater decreases in the MedDiet group (no significant differences between groups observed). However both groups lost $>1$ BMI point, whereas we aim to maintain weight at baseline levels. Other randomised trials have investigated the impact of the MedDiet on cardiovascular health outcomes; the Lyon Diet heart study was conducted with secondary CVD patients in France [11], and the Prevencíon con dieta Mediterránea study was conducted in high-risk participants free of disease in Spain [12]. The results of both trials showed benefits of the MedDiet to the populations studied, for prevention of stroke, secondary myocardial infarction and improved lipid profiles. Indeed a review of experimental studies investigating the MedDiet suggested the diet improves endothelial function and the lipid profile, but the only Australian study included in the review was a weight loss study [43]. Geographical and methodological differences in study design and recruitment limit the applicability of such findings to an Australian population. Whether these benefits would occur in an Australian population without weight loss is likely but unknown.

The secondary aim of the MedLey trial is to determine whether Australians can follow the MedDiet. Good adherence with the MedDiet underpins essentially any health benefits achieved - if for any number of cultural, genetic, environmental or other reasons the MedDiet was unacceptable in Australia, it would not be a useful intervention. One small study $(n=27)$ amongst Australian-born adults with Type 2 diabetes gave participants a traditional MedDiet for 12 weeks, and volunteers achieved high compliance to the diet [17]. However almost all (minimum of $70 \%$ ) of the diet was provided as pre-prepared foods. We piloted the MedDiet used in MedLey in a small group of elderly adults and found good adherence over a fortnight, and good general acceptance of the diet (Davis et al. 2015 under review), which was indicative that it could be adhered to over a longer period.
The collection of cardiometabolic and dietary data over 6 months in MedLey should provide information as to whether Australians can follow the MedDiet and whether there will be similar health benefits as seen in other countries. Future publications will detail major outcomes from MedLey and add understanding to whether the MedDiet should be promoted in Australia.

Trial status: Ongoing (data analysis).

\section{Consent for publication}

Not applicable.

\section{Abbreviations}

CVD: Cardiovascular disease; MedDiet: Mediterranean diet; MedLey: Mediterranean diet for cognitive and cardiovascular health in the elderly; DLQ: Diet and lifestyle questionnaire; hs-CRP: High sensitivity C-reactive protein; HabDiet: Habitual diet; BMI: Body mass index; TCD: Trans cranial Doppler; FMD: Flow mediated dilatation; WFR: Weighed food record; FFQ: Food frequency questionnaire; DEXA: Dual x-ray absorptiometry; LDQ: Lifetime diet questionnaire; FCl: Food craving inventory; CVR: Cerebrovascular responsiveness; MCA: Middle cerebral artery; LDL: Low-density lipoprotein; HDL: High density lipoprotein; FAs: Fatty acids; NATA: National association of testing authorities; CMBN-Cyt: Cytokenesisblock micronucleus-Cytome; NDI: Nuclear division index; PCR: Polymerase chain reaction; EVOO: Extra virgin olive oil; MDS: Mediterranean diet score.

\section{Competing interests}

None to declare.

\section{Authors' contributions}

CD participated in study conception and design, dietary formulation, data collection and drafted the manuscript. KM secured the funding with JB, CW and $\mathrm{JH}$, participated in study design, dietary formulation, and manuscript revision. JB participated in study design, dietary formulation and manuscript revision. JH and CW participated in dietary formulation and manuscript revision. VD participated in study design and drafted the manuscript. All authors read and approved the final manuscript.

\section{Acknowledgements}

The authors thank Professor Graham Giles of the Cancer Epidemiology Centre of The Cancer Council Victoria, for permission to use the Dietary Questionnaire for Epidemiological Studies (Version 2), Melbourne: The Cancer Council Victoria, 1996; Prof Adrian Esterman for assistance with statistical design; Prof. Michael Fenech, Dr Philip Thomas and Dr Paul Cavuoto for the DNA damage biomarkers assessment and $\mathrm{ApoE}_{4}$ genotyping; Louise Massie, Sansom Clinical Trials Co-ordinator; Dr Catherine Yandell and Mark Cutting for assistance with data collection, trial assistance and sample handling; Crystal Grant, Kathryn Dyer and Nerylee Watson for assistance with data collection and sample handling; dietitians Natalie Blanch and Kristina Petersen for help with dietetic consultations; and Alissa Knight for cognitive assessments. Finally, we would like to acknowledge the kind donations contributions from the following organisations: Cobram Estate for providing Australian extra virgin olive oil; Peanut Company of Australia for providing hi-oleic dry roast peanuts; The Grains \& Legumes Nutrition Council $^{\mathrm{TM}}$ for sourcing foods for the trial, Simplot Australia Pty. Ltd for providing Edgell canned legumes and John West tuna; Goodman Fielder Ltd for providing canola oil; and The Almond Board of Australia for providing almonds.

\section{Funding}

National Health and Medical Research Council grant.

\section{Author details}

${ }^{1}$ Alliance for Research in Exercise, Nutrition and Activity, University of South Australia, GPO Box 2471, Adelaide, South Australia 5001, Australia. ${ }^{2}$ School of Psychology, Social Work and Social Policy, University of South Australian, GPO Box 2471, Adelaide, South Australia 5001, Australia. ${ }^{3}$ School of Medicine and Pharmacology, University of Western Australia, 35 Stirling Highway, Perth, WA 6009, Australia. ${ }^{4}$ Faculty of Medicine, Nursing and Health Sciences, Flinders Centre for Innovation in Cancer, School of Medicine, GPO BOX 2100, 
Adelaide, South Australia 5001, Australia. ${ }^{5}$ CSIRO Food and Nutrition, Gate 13, Kintore Avenue, PO Box 10041, Adelaide, South Australia 5000, Australia.

Received: 12 October 2015 Accepted: 13 November 2015 Published online: 19 November 2015

\section{References}

1. Sofi F, Macchi C, Abbate R, Gensini GF, Casini A. Mediterranean diet and health status: an updated meta-analysis and a proposal for a literaturebased adherence score. Public Health Nutr. 2014;17(12):2769-82. doi:10.1017/S1368980013003169.

2. Australian Institute of Health and Welfare. Australia's health 2012. Canberra: AlHW; 2012. Report No:: AUS 156

3. Ross R. Mechanisms of disease: atherosclerosis - An inflammatory disease. N Engl J Med. 1999;340(2):115-26.

4. Aviram M, Fuhrman B. LDL oxidation by arterial wall macrophages depends on the oxidative status in the lipoprotein and in the cells: Role of prooxidant vs. antioxidant. Mol Cell Biochem. 1998;188:149-59.

5. Massaro M, Scoditti E, Carluccio MA, De Caterina R. Nutraceuticals and prevention of atherosclerosis: Focus on $\omega-3$ polyunsaturated fatty acids and Mediterranean diet polyphenols. Cardiovasc Ther. 2010;28:e13-9. doi:10.1111/j.1755-5922.2010.00211.x.

6. Fung $\Pi$, Rexrode KM, Mantzoros CS, Manson JE, Willett WC, Hu FB Mediterranean diet and incidence of and mortality from coronary heart disease and stroke in women. Circulation. 2009;119:1093-100. doi:10.1161/CIRCULATIONAHA.108.816736.

7. Panagiotakos DB, Pitsavos C, Stefanadis C. Dietary patterns: a Mediterranean diet score and its relation to clinical and biological markers of cardiovacsular disease risk. Nutr Metab Cardiovasc Dis. 2006;16:559-68. doi:10.1016/j.numecd.2005.08.006.

8. Bamia C, Trichopoulos D, Ferrari P, Overvad K, Bjerregaard L, Tjønneland A, et al. Dietary patterns and survival of older Europeans: The EPIC-Eldely study (European Prospective Investigation into Cancer and Nutrition). Public Health Nutr. 2007;10(6):590-8. doi:10.1017/S1368980007382487.

9. Buckland G, González CA, Agudo A, Vilardell M, Berenguer A, Amiano P, et al, Adherence to the Mediterranean diet and risk of coronary heart disease in the Spanish EPIC cohort study. Am J Epidemiol. 2009:170(12):1518-29. doi:10.1093/aje/kwp282.

10. Dilis V, Katsoulis M, Lagiou P, Trichopoulos D, Naska A, Trichopoulou A. Mediterranean diet and CHD: the Greek European prospective investigation into cancer and nutrition cohort. Br J Nutr. 2012;108:699-709. doi:10.1017/S0007114512001821.

11. de Lorgeril M, Salen P, Martin J-L, Monjaud I, Delaye J, Mamelle N. Mediterranean diet, traditional risk factors, and the rate of cardiovascular complications after myocardial infarction: final report of the Lyon diet heart study. Circulation. 1999; 99:779-85. doi:10.1161/01.CIR.99.6.779.

12. Estruch R, Ros E, Salas-Salvadó J, Covas M-I, Corella D, Arós F, et al. Primary prevention of cardiovascualr disease with a Mediterranean diet. N Engl J Med. 2013;368:1279-90. doi:10.1056/NEJMoa1200303.

13. National Heart Foundation of Australia. Position statement: antioxidants in food, drinks and supplements for cardiovascular health. Canberra: National Heart Foundation of Australia; 2010

14. National Heart Foundation of Australia. Position statement: dietary fats and dietary sterols for cardiovascular health. National Heart Foundation of Australia. 2009. http://heartfoundation.org.au/for-professionals/food-andnutrition/position-statements. Accessed 5 Feb 2015.

15. Noakes M, Barbara E. Position statement on the relationships between carbohydrates, dietary fibre, glycaemic index/glycaemic load and cardiovascular disease. National Heart Foundation of Australia. 2006 http://heartfoundation.org.au/for-professionals/food-and-nutrition/ position-statements. Accessed 5 Feb 2015.

16. Vincent-Baudry S, Defoort C, Gerber M, Bernard M-C, Verger P, Helal O, et al. The Medi-RIVAGE study: reduction of cardiovascular disease risk factors after a 3-mo intervention with a Mediterranean-type diet or a low-fat diet. Am J Clin Nutr. 2005;82:964-71.

17. Itsiopoulos C, Brazionis L, Kaimakamis M, Cameron M, Best J, O'Dea K, et al. Can the Mediterranean diet lower HbA1c in type 2 diabetes? Results from a randomized cross-over study. Nutr Metab Cardiovasc Dis. 2011;21:740-7. doi:10.1016/j.numecd.2010.03.005.

18. Kalbe E, Kessler J, Calabrese P, Smith R, Passmore A, Brand M, et al. DemTect: a new, sensitive cognitive screening test to support the diagnosis of mild cognitive impairment and early dementia. Int J Geriatr Psychiatry. 2004;19:136-43. doi:10.1002/gps.1042.

19. Altman DG, Bland JM. Treatment allocation by minimisation. BMJ. 2005;330: 843. doi:10.1136/bmj.330.7495.843.

20. Giles $\mathrm{G}$, Ireland P. Dietary questionnaire for epidemiological studies (Version 2). Melbourne: The Cancer Council Victoria; 1996.

21. Xinying PX, Noakes M, Keogh J. Can a food frequency questionnaire be used to capture dietary intake data in a 4 week clinical interveniton trial? Asia Pac J Clin Nutr. 2004;13(4):318-23.

22. Hosking D, Danthiir V, Nettelbeck T, Wilson C. Assessing lifetime diet: reproducibility of a self-administered, non qualitative FFQ. Public Health Nutr. 2010;14(5):801-8. doi:10.1017/\$1368980010003174.

23. White MA, Whisenhunt BL, Williamson DA, Greenway FL, Netemeyer RG. Development and validation of the food craving inventory. Obes Res. 2002;10(2):107-14

24. Knight A, Bryan J, Wilson C, Hodgson J, Murphy KJ. A randomised controlled intervention trial evaluating the efficacy of a Mediterranean dietary pattern on cognitive function and psychological wellbeing in healthy older adults: the MedLey study. BMC Geriatr. 2015;15:55. doi:10.1186/s12877-015-0054-8.

25. QualityMetric Incoporated and Medical Outcomes Trust. All rights reserved. SD-36V2 ${ }^{\mathrm{TM}}$ Health survey, standard. 1999.

26. Marteua TM, Bekker $\mathrm{H}$. The development of a six-item short form of the state scale of the Spielberger State-Trait Anxiety Inventory (STAI). Br J Clin Psychol. 1992;31:301-6.

27. Radloff LS. The CES-D scale: a self-report depression scale for research in the general population. Appl Psychol Meas. 1977;1(3):385-401.

28. Cohen S, Karmarck T, Mermelstein R. A global measure of perceived stress. J Health Soc Behav. 1983;24(4):385-96.

29. Parrot AC, Hindmarch I. The leeds sleep evaluation questionnaire in psychopharmacological investigations - a review. Psychopharmacology (Berl). 1980;71:173-9.

30. Stout M. Flow-mediated dilatation: a review of techniques and applications Echocardiography. 2009;26(7):832-41. doi:10.1111/j.1540-8175.2009.00927.x.

31. Barua AB, Kostic D, Olson JA. New simplified procedures for the extraction and simultaneous high-performance liquid chromatographic analysis of retinol, tocopherols and carotenoids in human serum. J Chromatogr B Biomed Sci Appl. 1993;617(2):257-64. doi:10.1016/0378-4347(93)80496-Q.

32. Tu W, Mühlhäusler B, Yelland L, Gibson R. Correlations between blood and tissue omega-3 LCPUFA staus following dietary ALA intervention in rats. Prostaglandins Leukot Essent Fatty Acids. 2013;88:53-60. doi:10.1016/.jplefa.2012.04.005.

33. Koch W, Ehrenhaft A, Griesser K, Pfeufer A, Muller J, Schomig A, et al. TaqMan systems for genotyping of disease-realted polymorphisms present in the gene encoding apolipoprotein E. Clin Chem Lab Med. 2002;40(11):1123-31. doi:10.1515/cclm.2002.197

34. Fenech M. Cytokinesis-block micronucleus cytome assay. Nat Protoc. 2007; 2(5):1084-104. doi:10.1038/nprot.2007.77.

35. Cawthorn RM. Telomere length measurment by a novel monochrome multiplex quantitative PCR method. Nucleic Acids Res. 2009;37:3. doi:10.1093/nar/gkn1027.

36. Lin J, Epel E, Cheon J, Kroenke C, Sinclair E, Bigos M, et al. Analyses and comparisons of telomerase activity and telomere length in human $\mathrm{T}$ and $\mathrm{B}$ cells: Insights for epidemiology of telomere maintenance. J Immunol Methods. 2010;352(1-2):71-80. doi:10.1016/j.jim.2009.09.012.

37. Norton K, Olds T. Anthropometrica. Sydney: UNSW Press; 1996

38. National Health and Medical Research Council, Department of Health and Ageing. Australian dietary guidelines: summary. Canberra: Department of Health and Ageing; 2013

39. Kromhout D, Keys A, Aravanis C, Buzina R, Fidanza F, Giampaoli S, et al. Food consumption patterns in the 1960s in seven countries. Am J Clin Nutr. 1989:49:889-94.

40. Bach-Faig A, Berry EM, Lairon D, Reguant J, Trichopoulou A, Dernini S, et al. Mediterranean diet pyramid today. Science and cultural updates. Public Health Nutr. 2011:14(12A):2274-84. doi:10.1079/PHN2004557.

41. National Health and Medical Research Council. Nutrient reference values for Australia and New Zealand; including recommended dietary intakes. Canberra: Department of Health and Ageing; 2006.

42. Norman GR, Sloan JA, Wyrwich KW. Interpretation of changes in health-related quality of life: the remarkable universality of half a standard deviation. Med Care. 2003:41(5):582-92. doi:10.1097/01.MLR.0000062554.74615.4C.

43. Serra-Majem L, Roman B, Estruch R. Scientific evidence of interventions using the Mediterranean diet: a systematic review. Nutr Rev. 2006;64(2):S27-47. doi:10.1301/nr.2006.feb.S27-S47. 